colorectal cancer patients are non-responsive and the 5-year survival rate for advanced disease is $<20 \%$. Immunotherapeutic response has been associated with select members of the microbiome in melanoma; however, the potential benefit in colorectal cancer and the underlying mechanisms remain unclear. We sought to determine how specific members of the intestinal microbiome affect anti-tumor immunity in colorectal cancer (CRC) in hopes of discovering novel treatments and revealing potential hurdles to current therapeutic response in CRC patients.

Methods We utilized a carcinogen-induced mouse model of CRC and colonized half of the tumor-bearing mice with Helicobacter hepaticus (Hhep) 7 weeks post AOM. Tumor number was assessed 12 weeks post AOM. We isolated lymphocytes from the lamina propria, colonic epithelium, mesenteric lymph nodes, and tumor(s) to track the spatial and transcriptional Hhep-specific and endogenous immune responses during tumor progression through 5' single cell RNAseq, flow cytometry, and immunofluorescence. In addition, we utilized $16 \mathrm{~S}$ sequencing and FISH to track Hhep colonization, location within the colon, and its impact on the surrounding microbiome.

Results We have found that rational modification of the microbiome of colon tumor-bearing mice through addition of a single bacteria, Hhep, led to tumor control or clearance and a significant survival advantage. Colonization led to the expansion of the lymphatic network and development of numerous peri- or intra-tumoral tertiary lymphoid structures (TLS) composed of Hhep-specific CD4 $\mathrm{T}$ follicular helper cells (TFH) as well as the bacteria itself. This led to an overall 'heating' of the tumor, wherein we saw an increase of CD4 T cell infiltration to the tumor core as well as an increase in CD103+ type 1 DC (cDC1) recruitment through increased chemokines such as CCL5 and XCL1. Hhep-specific TFH were both necessary and sufficient to drive TLS formation, increased immune invasion, and anti-tumor immunity.

Conclusions We have shown that addition of a single bacteria, Hhep, leads to a reduction in CRC tumor burden or clearance through lymphatic expansion, TLS formation, and remodeling of the tumor microenvironment, and that Hhepspecific $\mathrm{T}$ cells are required for tumor control. These studies suggest that rational modification of the microbiome and microbiome-specific $\mathrm{T}$ cells can positively impact anti-tumor immunity and may represent a unique immunotherapeutic target to turn resistant tumors into responsive tumors.

http://dx.doi.org/10.1136/jitc-2020-SITC2020.0678

\section{HIGH FIBER DIET MODIFIES GUT MICROBIOME, PROPIONATE PRODUCTION, INTRATUMOR IMMUNE RESPONSE AND IS ASSOCIATED WITH OUTCOME IN PATIENTS WITH LUNG CANCER TREATED WITH IMMUNE CHECKPOINT INHIBITORS}

Corentin Richard*, Myriam Benlaifaoui, Omar El Ouarzadi, Khoudia Diop, Antoine Desilets, Julie Malo, Wiam Belkaid, Andréanne Leblanc, Julien Lamontagne, Meriem Messaoudene, Arielle Elkrief, Bertrand Routy. CRCHUM, Montreal, Canada

Background The gut microbiome plays a key role in immune checkpoint inhibitors (ICI) efficacy and several strategies are currently being investigated to improve microbiome composition. The impact of a specific diet on microbiome modulation and clinical outcomes remains unknown. In this study, we assessed the effects of a high fiber diet on clinical outcomes as well as on microbiome composition, production of fecal metabolites, and intratumor immune infiltration in metastatic non-small cell lung cancer (mNSCLC) patients amenable to ICI.

Methods In this prospective study, 39 chemotherapy-refractory or naive patients with mNSCLC treated with ICI alone or in combination with chemotherapy completed a validated dietary survey. Based on the total fiber intake, patients were divided into high vs low fiber groups (HF vs LF). Objective response rate (ORR), progression-free survival (PFS) and overall survival (OS) were compared between both groups. In addition, fecal and tumor samples were collected prior to ICI initiation. Fecal metagenomic sequencing was performed and fecal short-chain fatty acids (SCFA) were measured by LC-MS/MS. Tumoral transcriptome profiling was performed through RNA sequencing to define differentially expressed pathways.

Results Baseline characteristics were well balanced between both groups, including body mass index (BMI) and PD-L1 status. Median PFS for the HF group was longer compared to the LF group (27.4 vs 12.6 months). Microbiome metagenomic profiling revealed higher baseline alpha diversity $(p=0.048)$ in the HF group compared to the LF group. Bifidobacterium, Alistipes, and Bacteroides salyersiae were enriched in the HF group while Fusobacterium was overrepresented in the LF group. SCFA measurement revealed that a high level of propionate correlated with a significantly longer OS (not reached vs 18.4. months, $\mathrm{p}=0.02$ ) in the entire cohort. Moreover, propionate levels were significantly higher in the HF vs LF group $(p=0.02)$. At the tumor level, RNA sequencing demonstrated a downregulation of DNA repair mechanisms and an upregulation of humoral and adaptive immune responses in the HF group.

Conclusions In this study, we demonstrated that a HF diet in patients with mNSCLC was associated with better clinical outcomes. Importantly, HF was associated with an enrichment of previously reported beneficial gut bacteria. Moreover, propionate correlated with longer OS and was increased in the HF group. This study provides further insights into how the diet can beneficially shift the microbiome composition and intratumor immune responses in patients with mNSCLC treated with ICI and this may lead to novel, dietary-geared therapeutic avenues in the oncomicrobiome arena.

Ethics Approval The study was approved by CRCHUM Institution,s Ethics Board, approval number 17.035.

http://dx.doi.org/10.1136/jitc-2020-SITC2020.0679

\section{AUTOMATED ION TORRENT BASED SOLUTION ENABLES ACCURATE GUT MICROBIOME QUANTIFICATION OF BACTERIAL SPECIES RELEVANT TO RESEARCH IN CANCER AND ITS RESPONSE TO IMMUNOTHERAPY}

Shrutii Sarda*, David Merrill, Heesun Shin, Anna McGeachy, Birgit Drews, Wing Lee, Rajesh Gottimukkala, Janice Au-Young, Fiona Hyland. Thermo Fisher Scientific, South San Francisco, USA

Background A low-cost targeted solution to profiling gut microbial diversity is sequencing of the 16S rRNA gene; however, it is often insufficient to gain species level resolution due to high homology across different bacteria. Therefore, we developed a first-of-its-kind targeted sequencing solution that supplements $16 \mathrm{~S}$ gene targets, with highly species-specific primers for a cohort of 73 bacteria associated with research in diabetes, cancer and its response to immunotherapy, 